\title{
GPRC5A is a potential oncogene in pancreatic ductal adenocarcinoma cells that is upregulated by gemcitabine with help from HuR
}

\author{
H Zhou ${ }^{1}$, AG Telonis ${ }^{1}$, Y Jing ${ }^{1}$, NL Xia ${ }^{2}$, L Biederman ${ }^{3}$, M Jimbo ${ }^{4}$, F Blanco ${ }^{4,5}$, E Londin ${ }^{1}$, JR Brody ${ }^{4}$ and I Rigoutsos ${ }^{\star, 1}$
}

GPRC5A is an orphan G-protein coupled receptor with an intriguing dual behavior, acting as an oncogene in some cancers and as a tumor suppressor in other cancers. In the pancreatic cancer context, very little is known about GPRC5A. By analyzing messenger RNA (mRNA) expression data from 675 human cancer cell lines and 10609 samples from The Cancer Genome Atlas (TCGA) we found that GPRC5A's abundance in pancreatic cancer is highest (cell lines) or second highest (TCGA) among all tissues and cancer types. Further analyses of an independent set of 252 pancreatic normal and cancer samples showed GPRC5A mRNA to be more than twofold upregulated in primary tumor samples compared with normal pancreas $\left(P\right.$-value $\left.<10^{-5}\right)$, and even further upregulated in pancreatic cancer metastases to various organs ( $P$-value $=0.0021$ ). Immunostaining of 208 cores ( 103 samples) of a tissue microarray showed generally low expression of GPRC5A protein in normal pancreatic ductal cells; on the other hand, in primary and metastatic samples, GPRC5A protein levels were dramatically increased in pancreatic ductal cells. In vitro studies of multiple pancreatic cancer cell lines showed that an increase in GPRC5A protein levels promoted pancreatic cancer cell growth and migration. Unexpectedly, when we treated pancreatic cancer cell lines with gemcitabine $\left(2^{\prime}, 2^{\prime}\right.$-difluorodeoxycytidine), we observed an increase in GPRC5A protein abundance. On the other hand, when we knocked down GPRC5A we sensitized pancreatic cancer cells to gemcitabine. Through further experimentation we showed that the monotonic increase in GPRC5A protein levels that we observe for the first $18 \mathrm{~h}$ following gemcitabine treatment results from interactions between GPRC5A's mRNA and the RNA-binding protein HuR, which is an established key mediator of gemcitabine's efficacy in cancer cells. As we discovered, the interaction between GPRC5A and HuR is mediated by at least one HuR-binding site in GPRC5A's mRNA. Our findings indicate that GPRC5A is part of a complex molecular axis that involves gemcitabine and HuR, and, possibly, other genes. Further work is warranted before it can be established unequivocally that GPRC5A is an oncogene in the pancreatic cancer context. Cell Death and Disease (2016) 7, e2294; doi:10.1038/cddis.2016.169; published online 14 July 2016

Pancreatic cancer is lethal and the fourth leading cause of cancer deaths in the United States with a 5-year overall survival rate of $6.7 \% .^{1}$ In 2014 , more than 46000 people were diagnosed with pancreatic cancer in the United States. Pancreatic ductal adenocarcinoma (PDAC) currently accounts for most of the diagnosed cases. Despite great efforts and very significant progress in elucidating the molecular events of pancreatic tumorigenesis, many of the details remain unknown. The disease's idiosyncratic attributes (e.g. cellular and molecular heterogeneity, extensive peritumoral stroma and unknown drug resistance mechanisms) have made it difficult to target both established (e.g. K-ras mutations) and more recently uncovered PDACspecific molecular events. ${ }^{2}$ To date, the majority of PDAC studies have focused on elucidating the impact of genetic mutations, the role of proteins, and the role of microRNAs (miRNAs) and their interactions with messenger RNAs (mRNAs). ${ }^{1,3}$

Recent research reports have suggested that the G-proteincoupled receptor, class $\mathrm{C}$, group 5 , member A or GPRC5A for short, may play important roles in a variety of settings. ${ }^{4}$ GPRC5A was first discovered in 1998 and became known initially as retinoic acid-induced gene $3(R A / 3)$ or retinoic acidinduced gene 1 (RAIG1). ${ }^{5}$ Very early experiments with multiple breast cancer cell lines linked the gene to the malignancy whereas siRNA knockdown in a single pancreatic cell line showed the induction of morphological changes. ${ }^{6}$ The available data to date suggest that the protein is very abundant in normal lung, normal liver, normal kidney, normal cerebellum, breast cancer, pancreatic cancer, etc. ${ }^{4,5}$ The protein

\footnotetext{
${ }^{1}$ Computational Medicine Center, Sidney Kimmel Medical College, Thomas Jefferson University, 1020 Locust Street Philadelphia, PA 19107, USA; ${ }^{2}$ Department of Neuroscience and The Farber Institute for Neuroscience, Thomas Jefferson University, 900 Walnut Street, Philadelphia, PA 19107, USA; ${ }^{3}$ Department of Pathology, Anatomy and Cell Biology, Sidney Kimmel Medical College, Thomas Jefferson University, 1020 Locust Street, Philadelphia, PA 19107, USA and ${ }^{4}$ Department of Surgery, The Jefferson Biliary and Related Cancer Center, Sidney Kimmel Medical College, Thomas Jefferson University, 1025 Walnut Street, Philadelphia, PA 19107, USA *Corresponding author: I Rigoutsos, Computational Medicine Center, Sidney Kimmel Medical College at Thomas Jefferson University, Jefferson Alumni Hall, \#M81, 1020 Locust Street, Philadelphia, PA 19107, USA. Tel: +1 215503 4219; Fax: +1 215503 0466; E-mail: isidore.rigoutsos@jefferson.edu

${ }^{5}$ Current address: 3819 Plum Hollow Dr. Houston, TX 77059, USA.

Abbreviations: CLIP-seq, cross-linking followed by immunoprecipitation and sequencing; HuR, human antigen R; PCRs, polymerase chain reaction; PDAC, pancreatic ductal adenocarcinoma; PL-5-GEM R, gemcitabine-resistant PL-5 cell line; RA/3, retinoic acid-induced gene 3; TCGA, The Cancer Genome Atlas; TMA, tissue microarrays; UTR, untranslated region; dCK, deoxycytidine kinase; mRNA, messenger RNA; miRNAs, microRNAs; ncRNAs, non-coding RNAs; qPCR, quantitative polymerase chain reaction

Received 14.12.15; revised 10.5.16; accepted 11.5.16; Edited by A Stephanou
} 
localizes primarily at the plasma membrane (see: The Human Protein Atlas, http://www.proteinatlas.org) and at cytoplasmic organelles, including the perinuclear vesicle, endoplasmic reticulum and the Golgi apparatus whereas, extracellularly, it has been identified in vesicular exosomes. ${ }^{4,5,7-10}$ GPRC5A has so far been shown to be dysregulated in many different human cancers as well as in other diseases. ${ }^{4,6,10-26}$ However, an increase in its abundance is not always associated with the cancer state: for example, in lung cancer GPRC5A levels are lower than in adjacent normal tissues; ${ }^{24}$ on the other hand, in several breast cancers GPRC5A levels are higher than in adjacent normal tissues. ${ }^{16,21}$

Mechanistically, GPRC5A was shown to interact with both non-coding RNAs (ncRNAs) and proteins. ${ }^{4,6,22-39}$ Post-translationally, GPRC5A protein can be phosphorylated by cyclindependent kinases in the cells, ${ }^{40-43}$ which suggests a role in cell cycle progression. The protein can also be $\mathrm{N}$-glycosylated and ubiquitinated. ${ }^{4,27,28,33,34,36,37}$ Dysregulation of GPRC5A was also shown to result in abnormal activation of $N F-k B$ signaling and STAT3 signaling. ${ }^{44}$ Recently, it was also shown that in breast cancer GPRC5A may be involved in DNA repair in cooperation with $\mathrm{BRCA} 1 .^{22}$ From a post-transcriptional regulation standpoint, however, very little is known.

ELAV-like protein 1, also known as 'human antigen R' or $\mathrm{HuR}$, is an RNA-binding protein that is encoded by the ELAVL1 gene. ${ }^{45,46}$ The HuR protein comprises three RNA-binding domains ${ }^{47,48}$ and has been found to bind preferentially AU-rich motifs in the $3^{\prime} U T R$ of mRNA transcripts, thereby increasing their stability. ${ }^{49,50}$ In terms of location, HuR is primarily found in the nucleus but translocates to the cytoplasm under the control of endogenous and exogenous factors. ${ }^{51} \mathrm{HuR}$ is known to regulate posttranscriptionally multiple genes and non-coding RNAs ${ }^{52-54}$ and to play key roles in human malignancies. ${ }^{55}$ Specifically in the pancreatic cancer context, HuR has been shown to regulate deoxycytidine kinase $(\mathrm{dCK})$, an enzyme that activates gemcitabine $\left(2^{\prime}, 2^{\prime}\right.$-difluorodeoxycytidine), thereby contributing, at least in part, to gemcitabine's efficacy in these cancer cells. ${ }^{56}$

In this report, we provide evidence that supports the hypothesis that GPRC5A acts as an oncogene in the pancreatic cancer context. Through a series of experiments with multiple pancreatic cancer cell lines, we examine the impact of GPRC5A overexpression on cell growth, colony formation ability and migration. In addition, we examine the role of the RNA-binding protein HuR, a key mediator of gemcitabine efficacy, in post-transcriptionally regulating GPRC5A and assess the ability of gemcitabine to modulate the abundance of GPRC5A in pancreatic cells. Lastly, we evaluate the impact of GPRC5A knockdown on the cancer cells' sensitivity to gemcitabine.

\section{Results}

Gene expression analyses of numerous cell lines and human samples show that GPRC5A mRNA levels in pancreatic cancer are among the highest and even further elevated in metastases. First, we analyzed publicly available RNA-seq data from 675 human cancer cell lines representing 17 human tissues. ${ }^{57}$ As can be seen in Figure 1a, GPRC5A mRNA is widely expressed across different tissues and cell line types. Its average expression is highest in pancreatic cancer cell lines. Then, we extended our analysis to the 10609 samples of the TCGA repository, which represent 33 different cancer types. As Figure 1b shows, GPRC5A mRNA is present abundantly across multiple cancers and exhibits the second highest average abundance in pancreatic ductal adenocarcinoma (PAAD). Having established that across all cancers GPRC5A is very abundant in pancreatic cancer, we analyzed previously reported microarray data ${ }^{2}$ (GEO accession number: GSE71729) and examined GPRC5A expression in normal pancreatic tissue $(n=46)$, primary pancreatic tumors $(n=145)$ and metastatic tumors $(n=61)$ using the $\mathrm{R}$ statistical computing environment. ${ }^{58}$ Figure $1 \mathrm{c}$ shows that compared with normal pancreas, GPRC5A is upregulated in primary tumors $\left(P\right.$-value $\left.<10^{-5}\right)$ and in pancreatic metastases $(P$-value $=$ $0.0021)$. As can be seen from the same figure, metastatic tumors had an even higher average expression of GPRC5A compared with primary tumors.

Immunohistochemical analyses show a dramatic increase of GPRC5A protein levels in primary PDAC samples and in several metastases. Having demonstrated with public data that GPRC5A mRNA is upregulated in both primary PDAC and metastases compared with normal pancreas, we sought to investigate whether GPRC5A protein levels changed in a concomitant manner. To this end, we stained 208 samples from 103 cases contained in a tissue microarray (PA2081a; US Biomax Inc, Rockville, MD, USA) with the same GPRC5A antibody (HPA007928; Sigma-Aldrich, St. Louis, MO, USA) that is used by the Human Protein Atlas (http://www.proteinatlas.org). We found that generally in both normal pancreatic tissue and adjacent normal pancreatic tissue from tumor, there was low to medium expression of GPRC5A protein in pancreatic ductal cells; however, in primary PDAC samples, GPRC5A protein levels increased dramatically (Figure $2 a$ and $b$ ). The high levels of GPRC5A protein in PDAC tumor cells persisted even when the cells metastasized to other organs like liver (Figure 2c). Supplementary Figure S1 shows additional examples of the stained samples from the tissue microarray.

GPRC5A mRNA and protein levels are high in multiple PDAC cell lines. We examined both GPRC5A mRNA and protein expression levels in six pancreatic cancer cell lines and in the normal pancreatic epithelial cell line hTERT-HPNE that is derived from the pancreas duct. As shown in Figure 2d, the level of GPRC5A mRNA in the six pancreatic cancer cell lines is statistically significantly higher compared with hTERT-HPNE. In concordance with the mRNA levels, GPRC5A protein levels are also increased in the six PDAC cell lines compared with hTERT-HPNE (Figure $2 e$ and Supplementary Figure S2A). Our quantitative polymerase chain reaction (qPCR) results are mirrored by the GPRC5A mRNA abundance in 38 pancreatic cell lines as gauged by next generation sequencing ${ }^{57}$ (Figure 1a). 


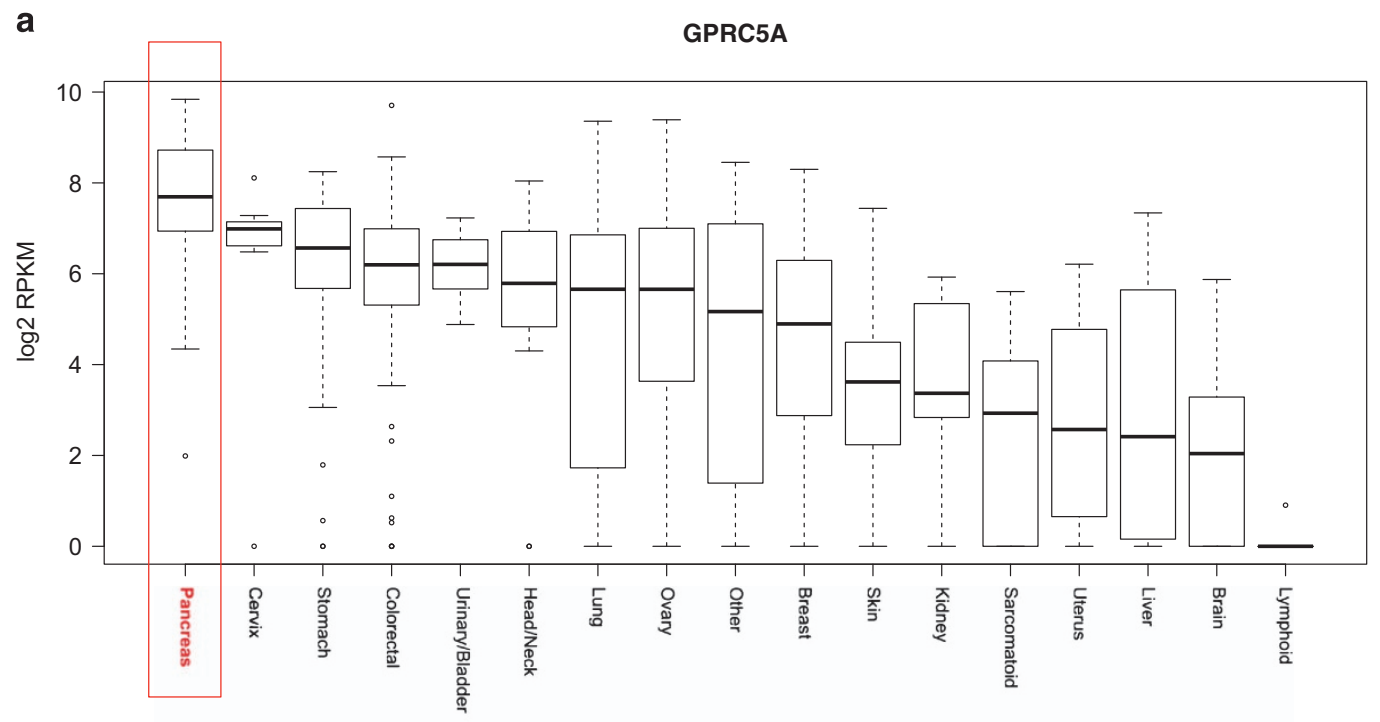

b

TCGA GPRC5A
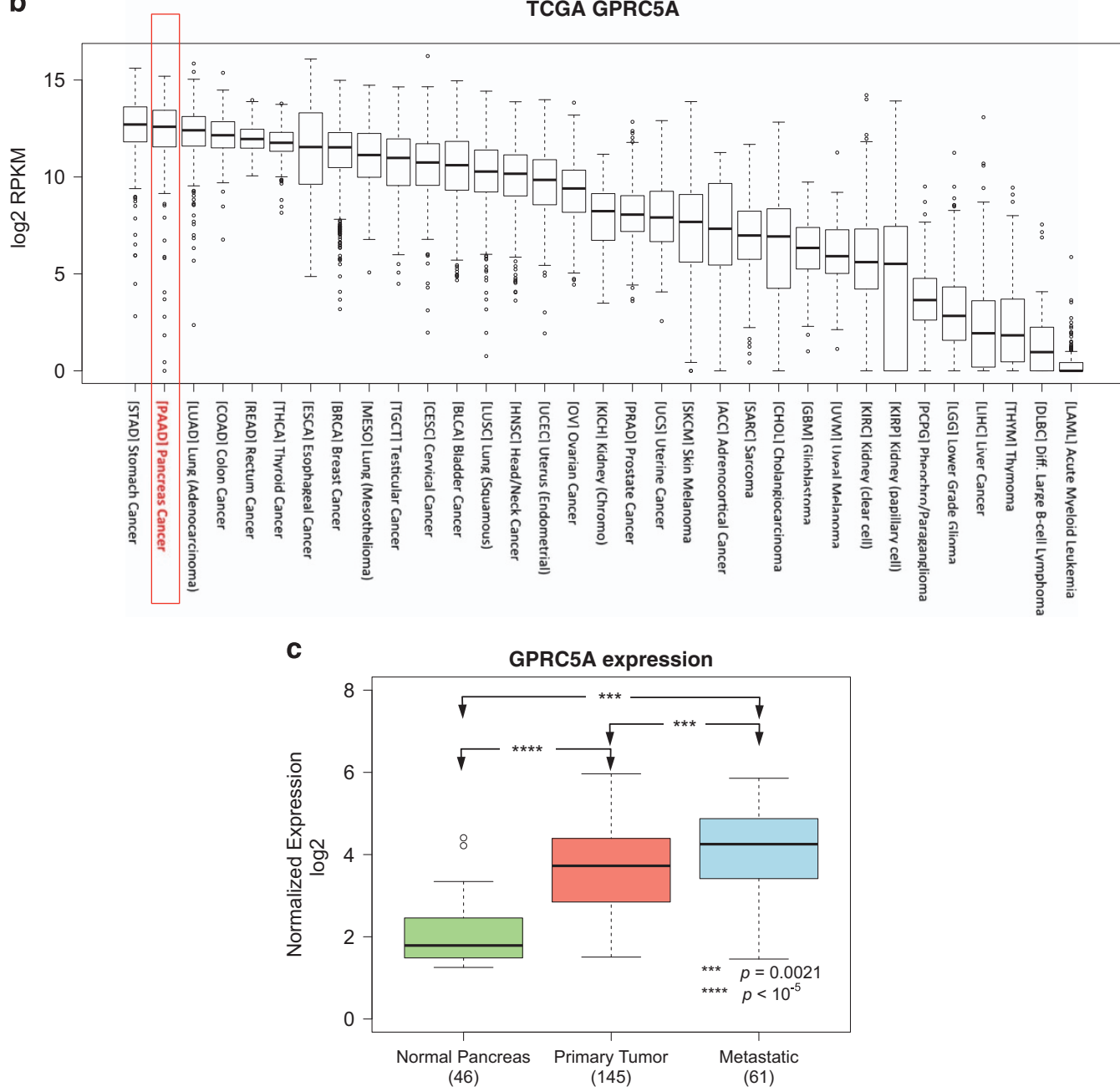

Figure 1 GPRC5A is highly expressed in pancreatic cancer cell lines and pancreatic cancer tissue. (a) RNA-seq datasets showing GPRC5A mRNA expression in different cancer cell lines. (b) RNA-seq data from TCGA showing GPRC5A mRNA expression in different cancers. (c) GPRC5A mRNA expression in normal pancreas, primary PDAC samples and metastases 
A

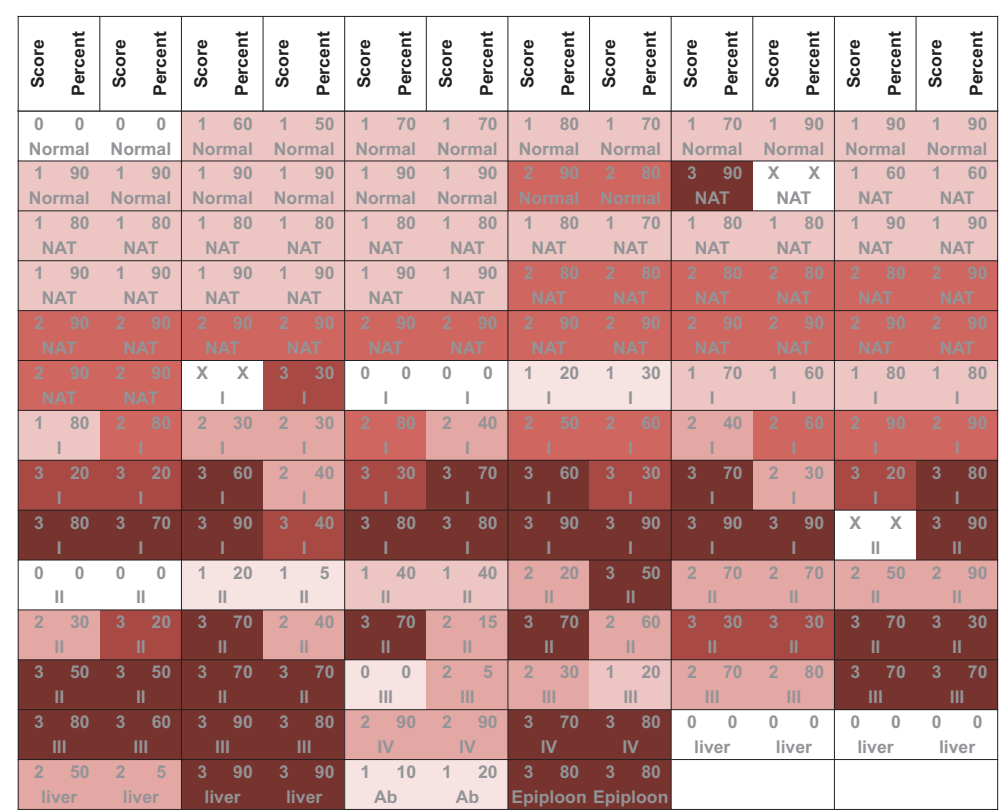

\begin{tabular}{l|lllllll}
\cline { 2 - 5 } Score/Percent & $0 / 0$ & $1 /<50$ & $1 />=50$ & $2 /<50$ & $2 />=50$ & $3 />50$ & 30 \\
\cline { 2 - 6 } & & &
\end{tabular}

\section{B}

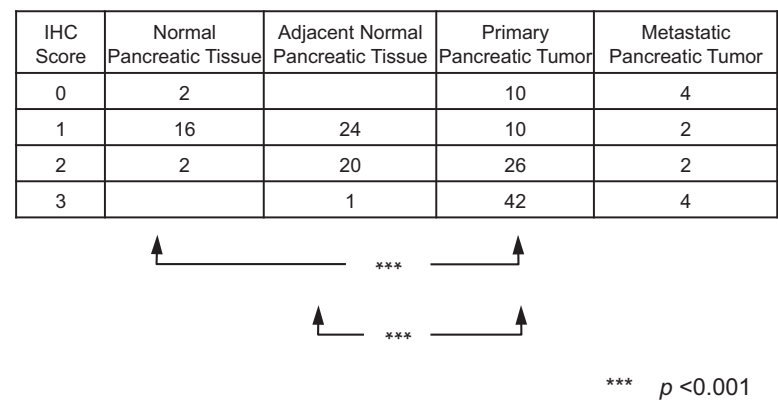

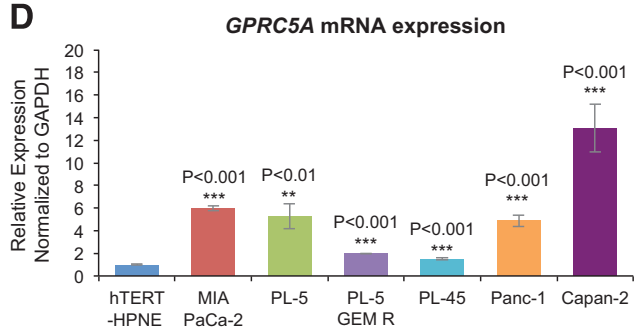

C GPRC5A expression in normal pancreatic tissues and tumors
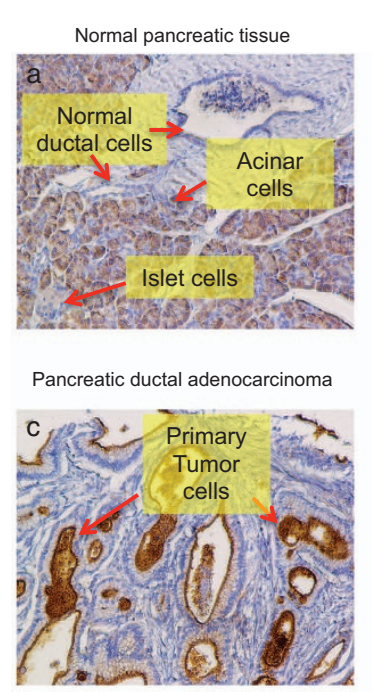

ancer adjacent norma

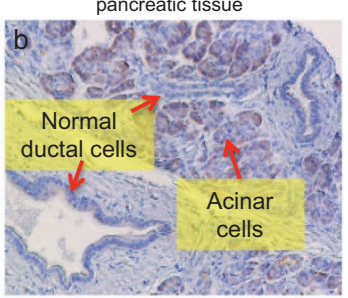

Pancreatic ductal adenocarcinoma metastasized to liver

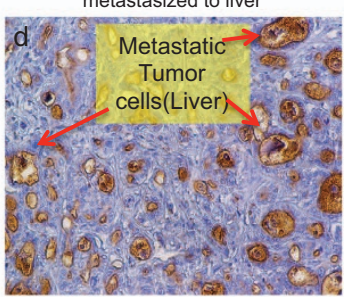

E

GPRC5A protein expression

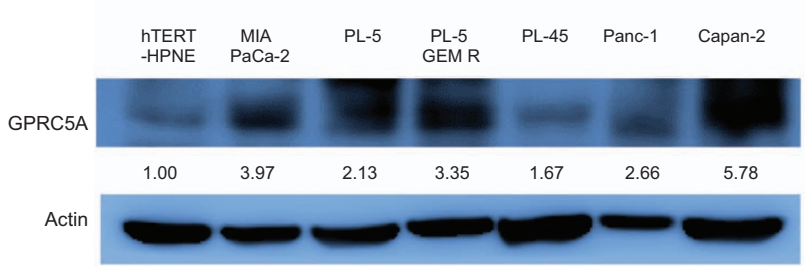

Figure 2 GPRC5A is upregulated in pancreatic ductal adenocarcinoma at both the mRNA level and the protein level. (a) Score table of TMA staining of 20 cores of normal pancreatic tissues, 42 cores of adjacent normal pancreatic tissues, 90 cores of primary pancreatic ductal adenocarcinoma and 12 cores of metastatic pancreatic ductal adenocarcinoma. (b) Summary of (a). (c) Examples of immunohistochemistry staining of GPRC5A in normal pancreatic tissues, primary PDAC samples and metastases. (d) GPRC5A mRNA expression in different pancreatic cell lines. (e) GPRC5A protein expression in different pancreatic cell lines. All numerical data are mean \pm S.D. ${ }^{\star} P<0.05$, ${ }^{* \star} P<0.01,{ }^{* \star *} P<0.001, n=3$ or as indicated. Ab, pancreatic ductal tumor metastases to abdominal cavity; Epiploon, pancreatic ductal tumor metastases to epiploon; Liver, pancreatic ductal tumor metastases to liver; NAT, adjacent normal tissue; Normal, normal pancreatic tissue; I,IIIIII, IV, pancreatic ductal cancer stages 
a

GPRC5A mRNA expression in hTERTHPNE cells

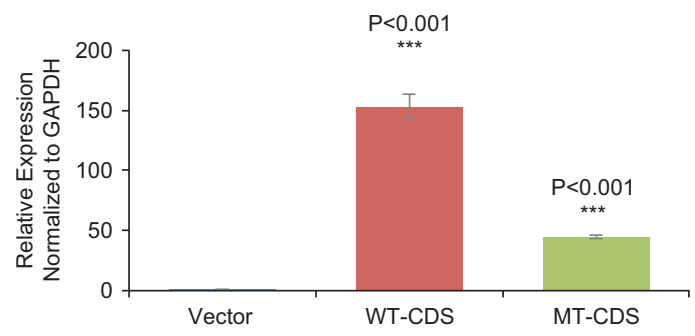

c

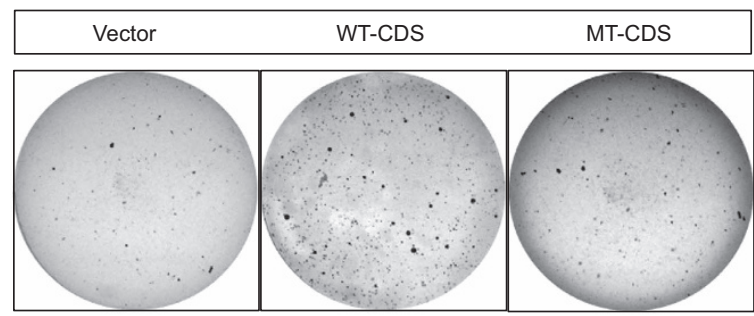

e
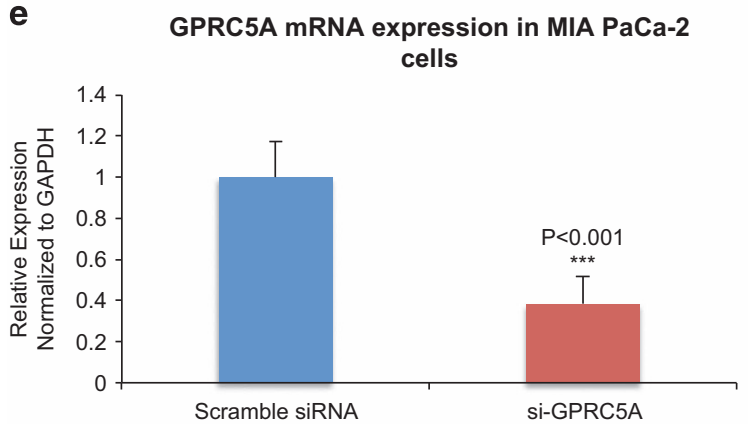

g

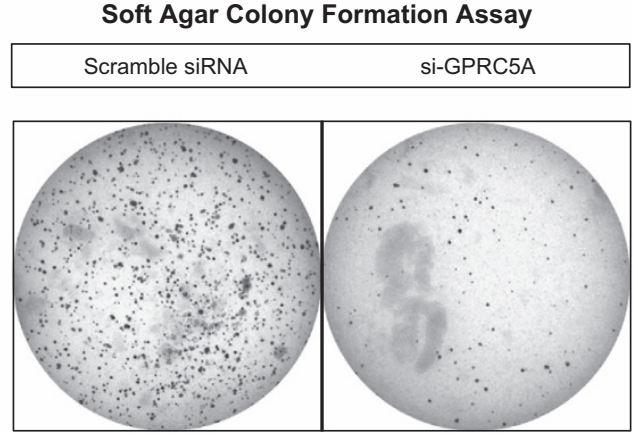

b

GPRC5A protein expression in hTERT-HPNE cells

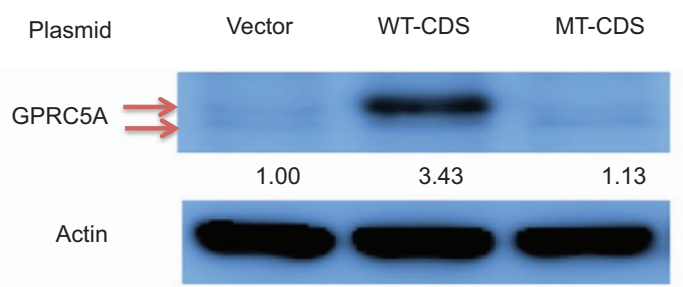

d

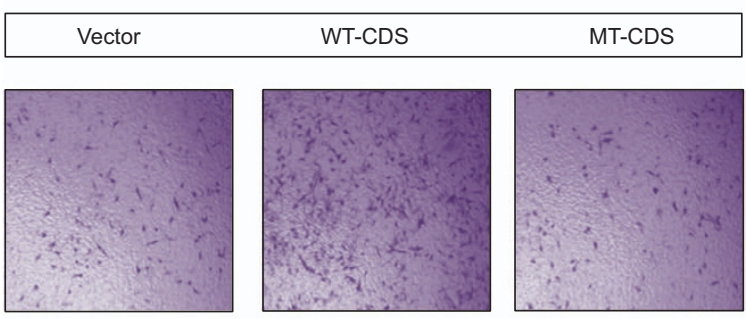

f

GPRC5A protein expression In MIA

PaCa-2 cells

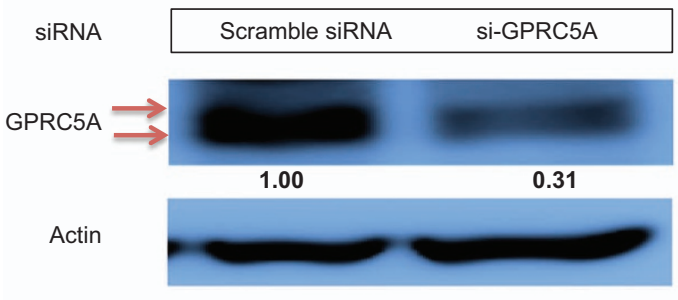

h

Cell Migration Assay

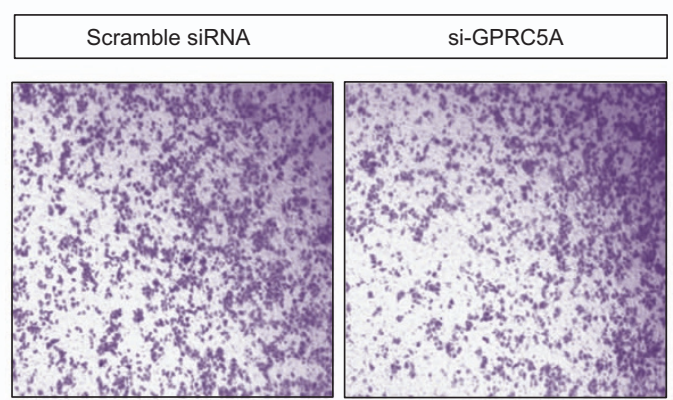

Figure 3 Ectopic expression of GPRC5A protein has an impact on pancreatic cells' colony formation ability and migration ability. (a) GPRC5A mRNA expression in hTERTHPNE cells after overexpression of GPRC5A WT-CDS or GPRC5A MT-CDS. (b) GPRC5A protein expression in hTERT-HPNE cells after overexpression of GPRC5A WT-CDS or GPRC5A MT-CDS. (c) Soft agar colony formation assay performed with hTERT-HPNE cells after overexpression of GPRC5A WT-CDS or GPRC5A MT-CDS. (d) Cell migration assay performed with hTERT-HPNE cells after overexpression of GPRC5A WT-CDS or GPRC5A MT-CDS. (e) GPRC5A mRNA expression in MIA PaCa-2 cells after treatment with siRNA. (f) GPRC5A protein expression in MIA PaCa-2 cells after treatment with siRNA. (g) Soft agar colony formation assay performed with MIA PaCa-2 cells after siRNA treatment. (h) Cell migration assay performed with MIA PaCa-2 cells after siRNA treatment. All numerical data are mean \pm S.D. ${ }^{*} P<0.05,{ }^{* \star} P<0.01,{ }^{* \star *} P<0.001, n=3$

Overexpression of GPRC5A promotes colony formation and migration in normal epithelial pancreatic cells. We overexpressed GPRC5A protein in hTERT-HPNE cells and examined its impact on the cells' ability to form colonies and to migrate. As controls, we alternately transfected hTERT-HPNE cells with a GPRC5A overexpression construct whose start codon was mutated, or, with the empty vector (Figures $3 a$ and $b$ and Supplementary Figure S2B). As demonstrated by the results shown in Figures $3 \mathrm{c}$ and $\mathrm{d}$ and in Supplementary Figures $\mathrm{S} 2 \mathrm{C}$ and $\mathrm{D}$, an increase in the abundance of GPRC5A protein promotes the ability of hTERT-HPNE cells to form colonies and to migrate. On the 
other hand, increasing the abundance of GPRC5A's mRNA has no such effect.

Inhibition of GPRC5A impairs colony formation and migration in PDAC cell lines. We designed an siRNA, si-GPRC5A (Figures $3 e$ and $f$ and Supplementary Figure S2E), and used it to examine whether inhibition of GPRC5A in PDAC cells could hinder colony formation and cell migration. SiGPRC5A (the targeted sequence is shown in Supplementary Table S1) specifically targets GPRC5A's 3'UTR. As our results in Figures $3 \mathrm{~g}$ and $\mathrm{h}$ and in Supplementary Figures S2F and G show, inhibition of GPRC5A in MIA PaCa-2 cells impaired the cells' colony formation in soft agar as well as reduced their migration ability. Moreover, when we inhibited GPRC5A in the pancreatic cancer cell lines Panc-1 and Capan-2 we could inhibit the cells' migration ability (Supplementary Figures S3A-D). Interestingly, we also found that inhibition of GPRC5A could hinder colony formation even in the normal epithelial pancreatic cell line hTERT-HPNE (Supplementary Figures S3E-H).

Inhibition of GPRC5A in PDAC cells diminishes cell survival following gemcitabine treatment. In light of the fact that GPRC5A knockdown reduced the ability of MIA PaCa-2 cells to form colonies and to migrate, we considered whether modulating the abundance of GPRC5A in parallel to gemcitabine treatment could affect the cells' sensitivity to the drug. We used si-GPRC5A to inhibit GPRC5A in MIA PaCa-2 cells, and then treated the cells with increasing concentrations of gemcitabine: we found that GPRC5A knockdown resulted in a decrease of cell survival and reduction of the cells' colony formation ability in the presence of gemcitabine at concentrations around $\mathrm{EC}_{50}$ (Figure 4). Experimentation with two more pancreatic cancer cell lines (Panc-1, PL-5) showed similar improvements in their gemcitabine sensitivity following GPRC5A knockdown (Supplementary Figures S4A-F). We also experimented with a gemcitabine-resistant PL-5 cell line (PL-5-GEM R): in this case, modulation of GPRC5A's abundance had an effect on the cells' response only when treated with high concentrations of gemcitabine (Supplementary Figures S4G-H). We also tested the effect of GPRC5A inhibition on MIA PaCa-2 cells' response to six more chemotherapy drugs (Supplementary Figure S5). With the exception of Trichostatin A, inhibition of GPRC5A in MIA PaCa-2 cells did not show significant effects on the cells' response to these drugs.

Gemcitabine treatment increases GPRC5A protein expression in MIA PaCa-2 cells through post-transcriptional events. We next treated MIA PaCa-2 cells with increasing concentrations of gemcitabine and examined whether treatment affected GPRC5A protein and mRNA levels. We found that upon gemcitabine exposure for $48 \mathrm{~h}$, GPRC5A protein expression increased monotonically with increasing gemcitabine concentrations eventually reaching a plateau at $\sim 100 \mathrm{nM}$ followed by a drop at $1 \mu \mathrm{M}$ (Figure $5 \mathrm{a}$ and Supplementary Figure S6A). Quantitation of GPRC5A mRNA levels revealed again two distinct regimes but they were different compared with those of the protein levels: there was little increase of mRNA abundance at lower gemcitabine concentrations followed by dramatically increases in mRNA abundance at higher concentrations (Figure 5b). Similar result was also shown in PL-5 cells (Supplementary Figures S6B-D).

This complementary behavior suggested the existence of post-transcriptional regulatory events that are induced by gemcitabine. The absence of changes in mRNA abundance in the regime of concentrations where protein expression increased suggested the possibility of transcript stabilization. On the other hand, the marked increase of GPRC5A mRNA at higher gemcitabine concentrations suggested a different mode of modulation that could result from transcriptional as well as post-transcriptional changes.

In recent work, we showed that miR-103a-3p interacts with the 5'-UTR of GPRC5A mRNA, thereby decreasing the abundance of both mRNA and protein. ${ }^{59}$ To examine the possibility of an miRNA-mediated post-transcriptional contribution to the observed mRNA abundance changes across different gemcitabine concentrations, we quantitated the corresponding levels of miR-103a-3p. As Figure $5 c$ shows, miR-103a-3p levels increase at a high gemcitabine concentration (200-1000 nM), yet remains largely constant at lower concentrations $(10-100 \mathrm{nM})$. There is lack of concordance between the miRNA and the mRNA and protein level changes shown in Figures $5 \mathrm{a}$ and $\mathrm{c}$ at a low concentration of gemcitabine treatment. The findings suggest that much of the observed modulation of GPRC5A protein abundance at low gemcitabine concentrations is not due to miR-103a-3p.

There are several possibilities that might underlie the apparent mRNA stabilization suggested by the increase of protein abundance at lower gemcitabine concentrations (Figure 5a), an increase that occurs without evident changes of mRNA at the same concentrations (Figure 5b). The apparent dependence on gemcitabine made the RNAbinding protein HuR (a.k.a. ELAVL1) a conspicuous candidate. Indeed, previous studies have shown that in pancreatic cells HuR is involved in post-transcriptionally stabilizing the translation of many important proteins. ${ }^{60,61}$ Specifically for our context, HuR has been shown to be a key mediator of gemcitabine's efficacy in cancer cells through its stabilization of dCK mRNA. ${ }^{56}$ We thus hypothesized HuR's involvement in mediating the observed behavior. To investigate this hypothesis, we carried out an HuR ribonucleoprotein immunoprecipitation (RIP) assay in MIA PaCa-2 cells. As Figure $5 d$ and Supplementary Figure S6E show, GPRC5A mRNA is bound by HuR, as is dCK (RIP positive control). When we knocked down HuR by SiRNA, the response of GPRC5A protein expression to the lower concentrations of gemcitabine was quashed (Supplementary Figures S7A and B), particularly in the 40-100 nM regime.

HuR post-transcriptionally regulates GPRC5A in MIA PaCa-2 cells. Having shown that HuR directly interacts with GPRC5A's mRNA (Figure $5 d$ ), we sought to examine the possibility that HuR underlies the changes in GPRC5A protein expression levels that we observed following gemcitabine exposure. We first treated MIA PaCa-2 cells with $400 \mathrm{nM}$ of gemcitabine and quantitated mRNA expression levels at several time points. As Figure 5 e shows, early on 

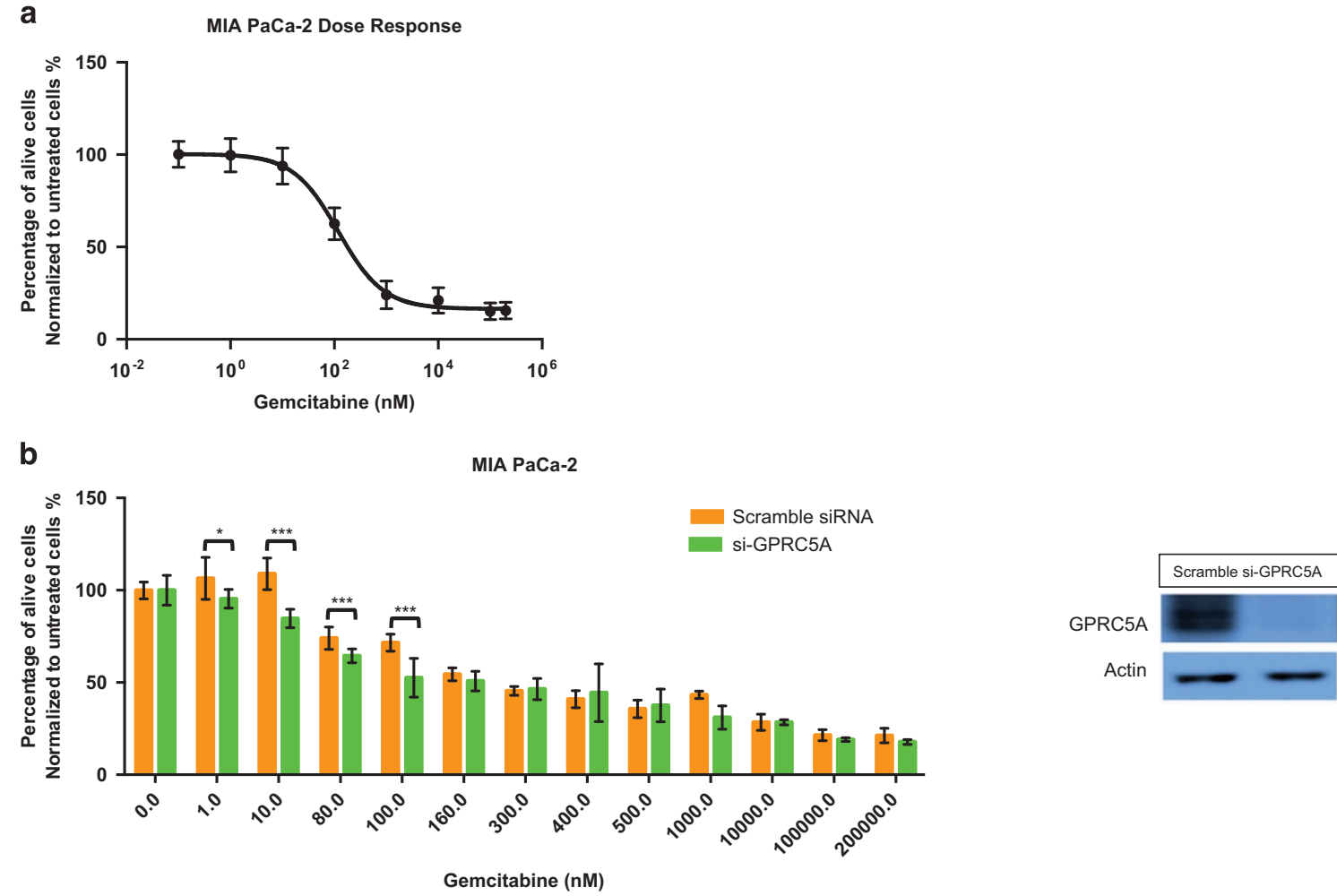

C

MIA PaCa-2

Quantification of MIA PaCa-2 colonies
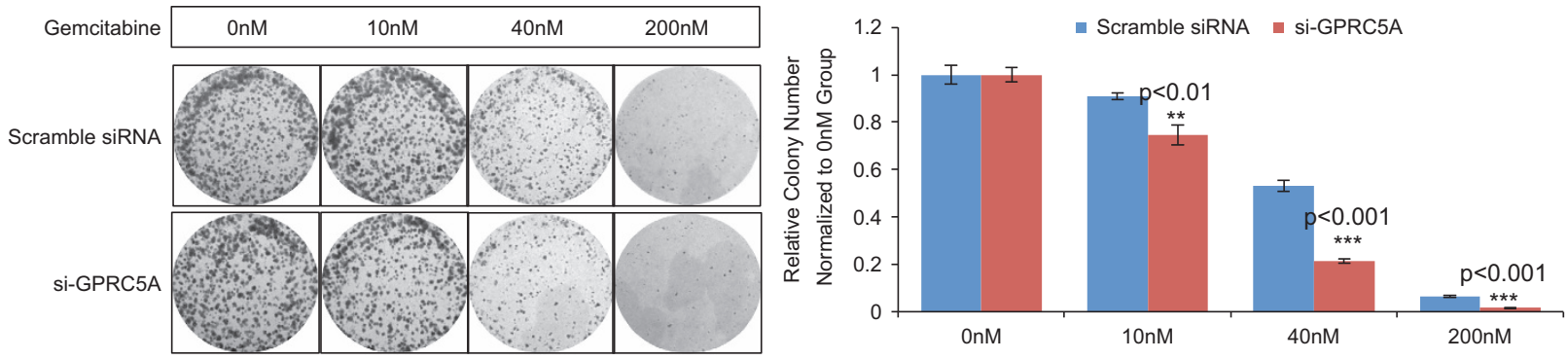

Figure 4 Knockdown of GPRC5A enhances pancreatic cancer cells' death in face of gemcitabine treatment. (a) MIA PaCa-2 cells' response to different concentrations of gemcitabine treatment. (b) Knockdown of GPRC5A in MIA PaCa-2 cells enhanced cells' death following gemcitabine treatment. (c) Knockdown of GPRC5A in MIA PaCa-2 cells reduced cells' colony formation ability when treated with gemcitabine. All numerical data are mean \pm S.D. ${ }^{*} P<0.05,{ }^{* *} P<0.01,{ }^{* * *} P<0.001, n=3$

(from 0 to $18 \mathrm{~h}$ ), GPRC5A mRNA levels remained unchanged whereas the abundance of GPRC5A protein level increased markedly as a function of time (Figure $5 f$ and Supplementary Figure S7C). During this time interval, even though the total amount of available HuR protein did not change significantly, HuR protein's cytoplasmic levels increased (Figure $5 f$ and Supplementary Figures S7C and D). ${ }^{60}$

Using a RIP assay, we showed that between 0 and $18 \mathrm{~h}$, the interaction between HuR protein and GPRC5A mRNA increased (Figure $5 \mathrm{~g}$ ) - note that the transcriptional abundance of GPRC5A remains unchanged during this time interval (Figure 5e). Thus, we conclude that HuR contributes to the observed increase in GPRC5A protein levels through mRNA stabilization. Forty-eight hours following gemcitabine treatment, there is significant upregulation of GPRC5A transcription (Figure 5e) and a concomitant increase in
GPRC5A protein abundance (Figure $5 f$ and Supplementary Figure S7A) and this occurs in the absence of mRNA stabilization by HuR, as evidenced by the HuR RIP assay of Figure $5 \mathrm{~g}$.

HuR binds at least one location in GPRC5A's 3'-UTR. In order to identify HuR's sites of interaction with GPRC5A's mRNA to pursue experimentally, we analyzed previously reported HuR CLIP-seq data and identified four such candidate sites (see Materials and Methods). We cloned separately each of the four sites (labeled 1, 2, 3 and 4). We also cloned the segment containing all four sites labeled as 'composite site' (Figure 6a). Figure 6b shows the results of luciferase assays using these five constructs. As can be seen, knockdown of HuR by siRNA reduced luciferase signal for the constructs containing sites 2 and 4 , and for the 
'composite site'; no significant change was observed for the constructs containing sites 1 and 3 , respectively. Upregulation of HuR via an HuR overexpression vector increased luciferase signal in the case of sites 1 and 4 , and of the 'composite site'; no significant change was observed in the case of sites 2 and 3 . PIM1 served as a positive control. ${ }^{62}$ a

GPRC5A protein expression in MIA PaCa-2 cells

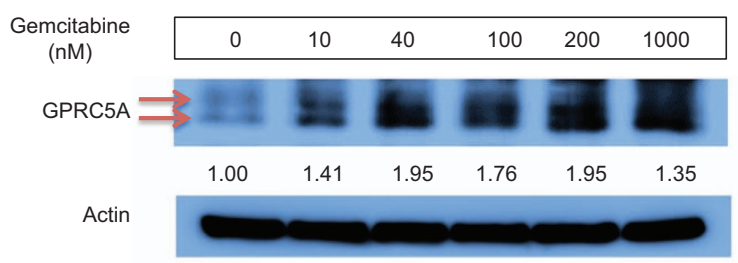

C

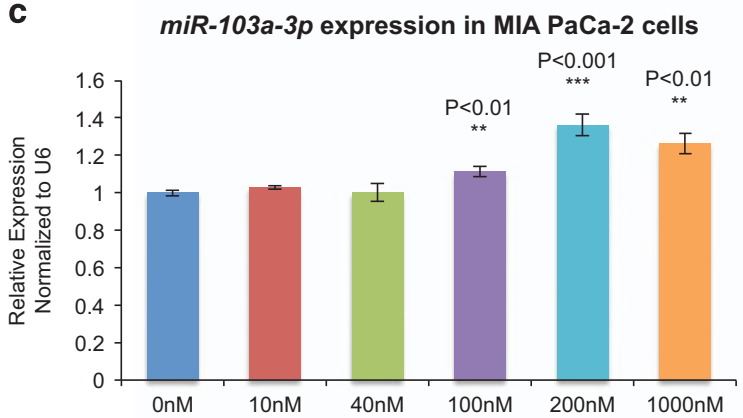

e GPRC5A mRNA expression in MIA PaCa-2 cells

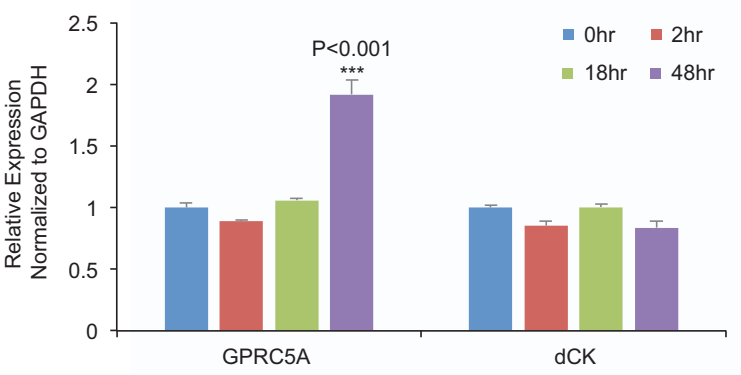

b

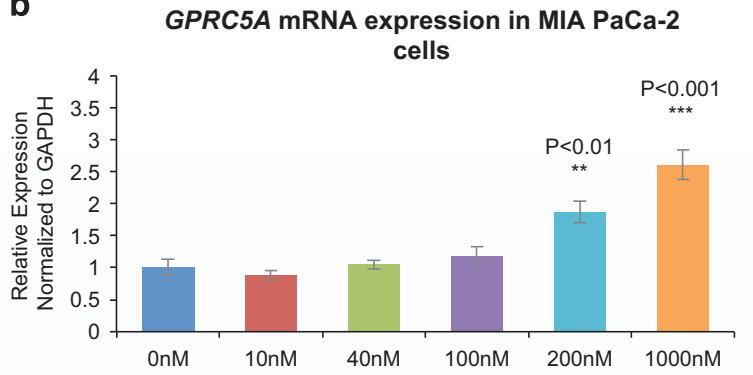

d

HuR RIP Assay

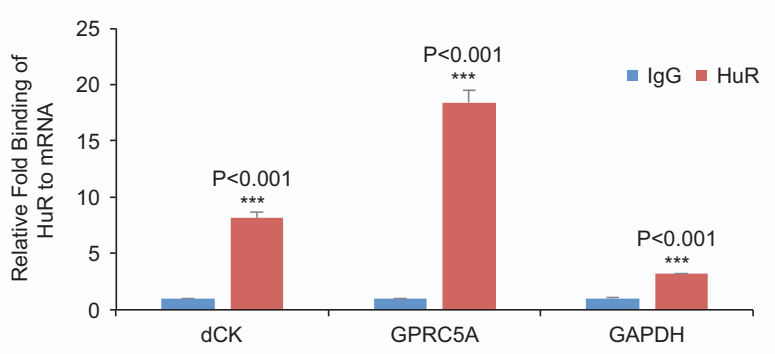

f

GPRC5A protein expression in MIA PaCa-2 cells

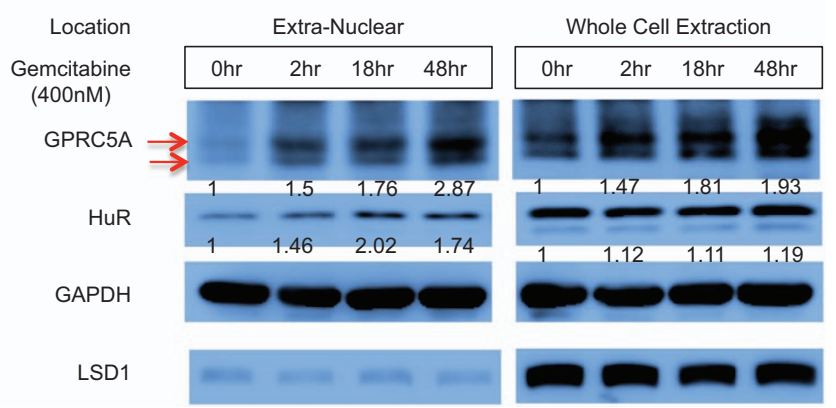

g

HuR RIP assay

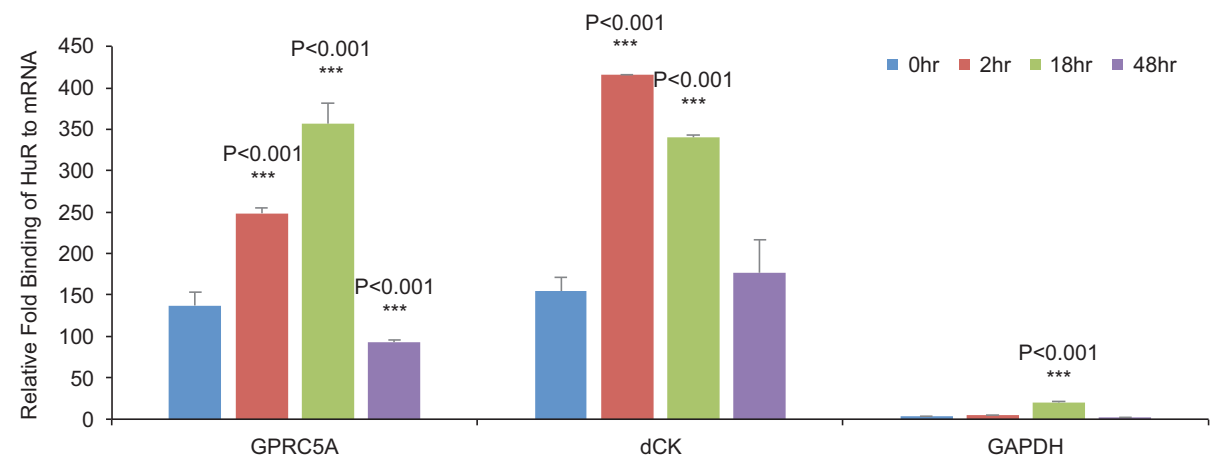

Figure 5 Gemcitabine treatment enhances GPRC5A expression in MIA PaCa-2 cells due to HuR in the early stage. (a) GPRC5A protein expression in MIA PaCa-2 cells after $48 \mathrm{~h}$ of treatment with gemcitabine at different concentrations. (b) GPRC5A mRNA expression in MIA PaCa-2 cells after $48 \mathrm{~h}$ treatment of gemcitabine in different concentrations. (c) miR-103a-3p expression in MIA PaCa-2 cells after $48 \mathrm{~h}$ of treatment with gemcitabine at different concentrations. (d) HuR RIP assay in MIA PaCa-2 cells. (e) GPRC5A mRNA expression in MIA PaCa-2 cells after $400 \mathrm{nM}$ gemcitabine treatment for $0,2,18$ and $48 \mathrm{~h}$. (f) GPRC5A protein and HuR protein levels in the cytoplasm or the whole-cell lysate of MIA PaCa-2 cells after $400 \mathrm{nM}$ gemcitabine treatment for 0, 2, 18 and $48 \mathrm{~h}$. (g) HuR RIP assay performed in MIA PaCa-2 cells after $400 \mathrm{nM}$ gemcitabine treatment for $0,2,18$ and 48 h. All numerical data are mean \pm S.D. ${ }^{*} P<0.05,{ }^{* \star} P<0.01,{ }^{* \star *} P<0.001, n=3$ 
a

Schematic representative of potential HuR binding sites

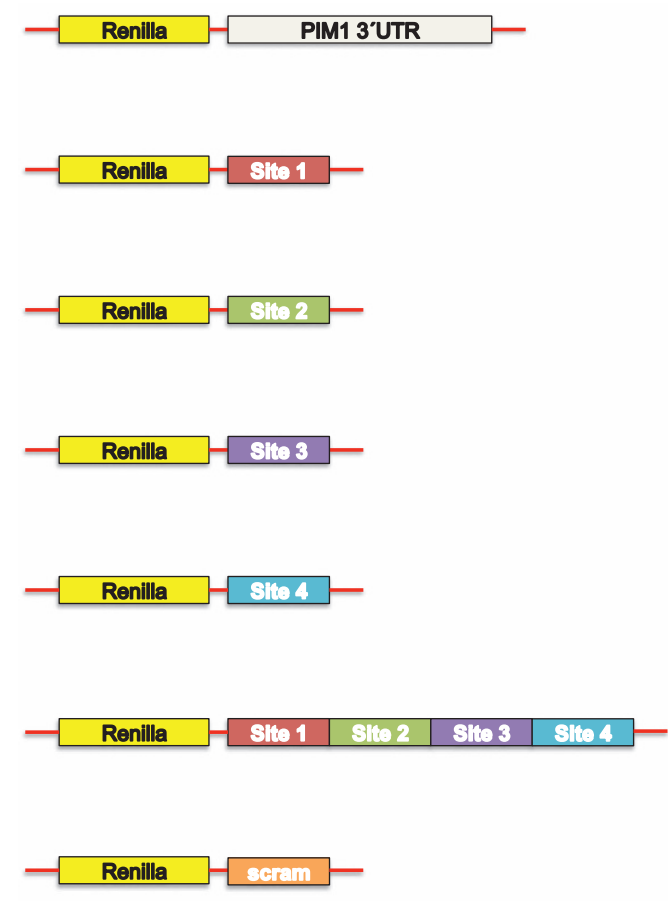

b

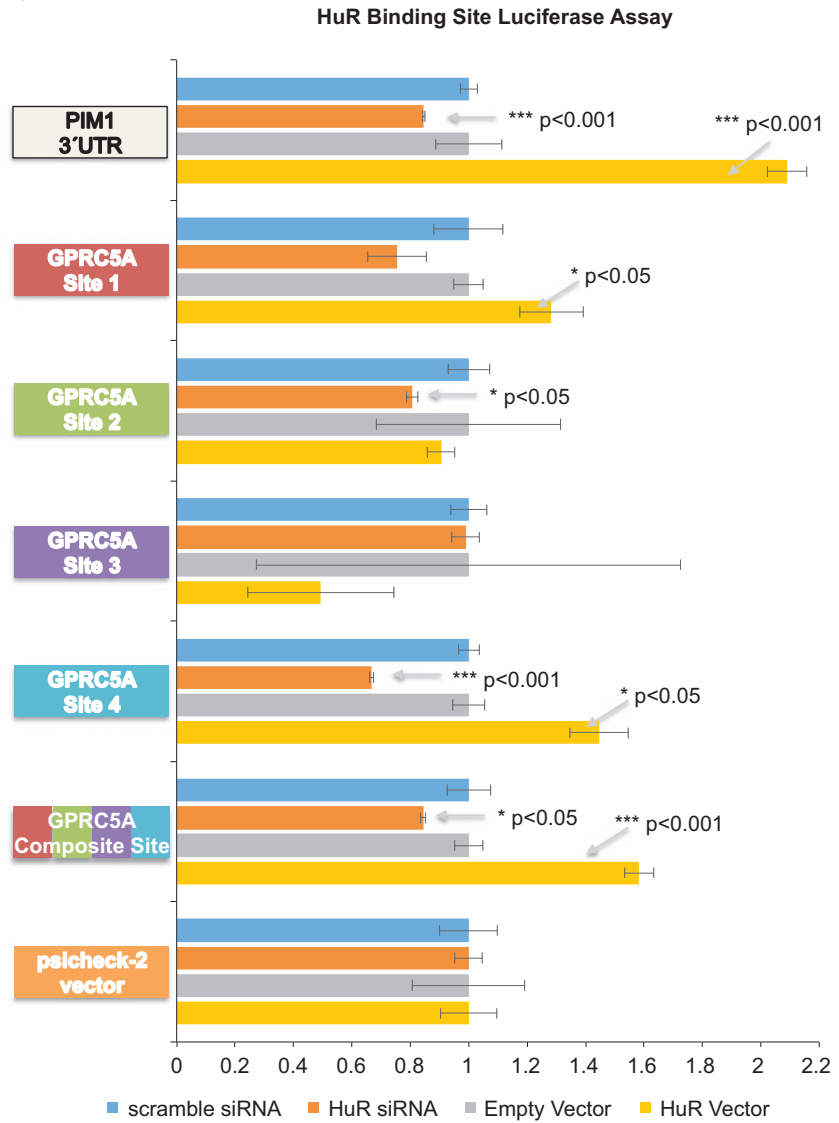

Figure 6 GPRC5A is directly regulated by HuR. (a) Schematic representative of potential HuR-binding sites. (b) HuR-binding site luciferase assay. Luciferase assay is performed in MIA PaCa-2 cells with psicheck-2 plasmid inserted with predicted HuR-binding sites. PIM1 is used as a positive control. ${ }^{62}$ All numerical data are mean \pm S.D. ${ }^{\star} P<0.05,{ }^{\star \star} P<0.01,{ }^{* \star *} P<0.001, n=3$

\begin{abstract}
Knockdown of GPRC5A could induce cell apoptosis, with or without gemcitabine treatment. Our further analyses of GPRC5A on MIA PaCa-2 cells' response to gemcitabine showed that knockdown of GPRC5A enhanced cell apoptosis without gemcitabine treatment; also, that administration of gemcitabine further enhanced pancreatic cancer cell apoptosis (Supplementary Figures S9A and B). At the molecular level, we found that knockdown of GPRC5A inhibited caspase 3 activation and increased accumulation of $\gamma \mathrm{H} 2 \mathrm{AX}$, a marker of DNA damage (Supplementary Figures S9C and D). Gemcitabine treatment could induce caspase 3 activation, as well as $\gamma \mathrm{H} 2 \mathrm{AX}$ accumulation in the control group. In the si-GPRC5Atreated group, gemcitabine failed to activate caspase 3 but still resulted in $\gamma \mathrm{H} 2 \mathrm{AX}$ accumulation.
\end{abstract}

\section{Discussion}

As the expression levels of GPRC5A in normal tissues vary very widely across tissues (from highly expressed in lung and colon to lowly expressed lines and pancreas), ${ }^{4}$ it is reasonable to posit that GPRC5A may be playing different roles in each of these contexts. This is further corroborated by previous reports of GPRC5A acting as a tumor suppressor in lung ${ }^{24}$ whereas in, for example, stomach and liver, increased
GPRC5A levels are associated with poor prognosis in patients with gastric cancer or hepatocellular carcinoma, respectively. ${ }^{20,26}$ In pancreas, previous research showed that GPRC5A was upregulated in the cancer state, ${ }^{6}$ but studies of its roles in pancreatic cancer have been limited.

In this report, we present evidence that in multiple pancreatic cancer cell lines the levels of GPRC5A mRNA and protein are higher compared with the normal pancreatic epithelial cell line hTERT-HPNE. We also show that in actual pancreatic tumor samples GPRC5A protein levels were dramatically higher than in normal pancreatic ductal cells.

When we overexpress GPRC5A in hTERT-HPNE the cells' ability to form colonies and to migrate compared with control is enhanced. Conversely, when we inhibit GPRC5A in pancreatic cancer cell lines such as MIA PaCa-2, Panc-1 and Capan-2 (Figures $3 e$ and $h$ and Supplementary Figures S2E-G and S3A-D) and in normal pancreatic cell line such as hTERTHPNE (Supplementary Figures 3E-H) we decrease the cells' ability to form colonies and we hinder migration.

Additionally, we are able to establish GPRC5A's participation in the pancreatic cancer cells' response to gemcitabine treatment. Specifically, increasing concentrations of gemcitabine lead to concomitant increases in GPRC5A protein levels. From a temporal standpoint, we show that for the first $18 \mathrm{~h}$ 
following gemcitabine treatment, GPRC5A protein levels increase monotonically but are solely the result of posttranscriptional events. At $48 \mathrm{~h}$ following exposure to gemcitabine, we observe a marked increase in GPRC5A mRNA levels. Gemcitabine treatment could induce apoptosis in pancreatic cancer cells (Supplementary Figures S9A and B) that was enhanced when we inhibited GPRC5A with an siRNA (Supplementary Figures S9A and B, Figures $4 \mathrm{a}-\mathrm{C}$ and Supplementary Figures S4A-H). In addition, our results showed that inhibition of GPRC5A could also sensitize pancreatic cancer cells to Trichostatin A (Supplementary Figure S5C). Additionally, we found that inhibition of STAT3 or NFkB activation could also sensitize pancreatic cancer cells to gemcitabine (Supplementary Figures S9E-I).

In previous studies, we showed that GPRC5A is regulated post-transcriptionally by miR-103a-3 $\mathrm{p}^{59}$ and, presumably, by other currently unknown miRNAs. In the present study, we present another dimension of post-transcriptional regulation of GPRC5A via its interaction with the RNA-binding protein HuR. Using luciferase assays, we demonstrate that HuR binds at least one site in the $3^{\prime}$-UTR of GPRC5A. Possibly more HuRbinding sites exist elsewhere in GPRC5A's rather long mRNA. We show that following the cellular stress caused by gemcitabine treatment, HuR protein translocates to the cytoplasm where it binds GPRC5A's mRNA and leads to a monotonic increase in GPRC5A protein levels for at least $18 \mathrm{~h}$ (Supplementary Figure S7D). By $48 \mathrm{~h}$ following gemcitabine exposure, the association of HuR and GPRC5A mRNA returns to background levels and post-transcriptional control of GPRC5A's mRNA decreases. MiR-103a-3p abundance, a known regulator of GPRC5A, ${ }^{59}$ does not change appreciably compared with $18 \mathrm{~h}$ (Supplementary Figure S7F). However, cytoplasmic GPRC5A protein levels increase even further at $48 \mathrm{~h}$ following gemcitabine exposure, the result of a transcriptional increase of GPRC5A mRNA in the cells.

In addition to gemcitabine, GPRC5A expression is affected following treatment with either 5-FU or Oxaliplatin. Interestingly, 5-FU treatment upregulates both GPRC5A mRNA and protein level at $48 \mathrm{~h}$ after administration (Supplementary Figures S8A-C) whereas Oxaliplatin has an effect on GPRC5A mRNA and protein expression as early as $24 \mathrm{~h}$ following treatment (Supplementary Figures S8D-F). These findings suggest that other factors may be involved in regulating GPRC5A expression in the presence of chemical stressors.

In summary, we presented evidence that suggests that GPRC5A participates in a complex set of interactions in the pancreatic cancer context (Figure 7). We also show that a codependent regulation of GPRC5A and HuR is accented in a time-dependent manner when cells are treated with gemcitabine. By potentially exploiting these interactions via targeting of GPRC5A mRNA we can increase the death rate of pancreatic cancer cells following treatment by gemcitabine or Trichostatin A. The increased abundance of GPRC5A in human cancer cell lines, TCGA cancer samples, and pancreatic cancer primary tumors and metastases, and the additional evidence we presented above, namely GPRC5A's interactions with HuR and gemcitabine as well as other potential chemo-drugs, suggest a possible pro-oncogenic role for this gene. Further studies of GPRC5A are warranted as it could prove to be a new candidate target for developing an alternative strategy for treating pancreatic cancer.

\section{Materials and Methods}

Cell culture. The MIA PaCa-2, hTERT-HPNE, PL-5, PL-45, Capan-2 and Panc-1 cell lines were obtained from American Type Culture Collection (Rockville, MD, USA). The gemcitabine-resistant PL-5-GEM $R$ line was generated in the Brody laboratory (Jimbo et al., unpublished). All cells, except PL-5-GEM R, were grown in DMEM medium (Fisher Scientific, Pittsburgh, PA, USA) supplemented with $10 \%$ fetal bovine serum (Life Technologies, Carlsbad, CA, USA), 1\% penicillin and streptomycin (Fisher Scientific), and $1 \%$ glutamine (Fisher Scientific) at $37^{\circ} \mathrm{C}$ in a humidified atmosphere containing $5 \% \mathrm{CO}_{2}$. PL-5 GEM R cells were grown in the above conditions with a supplement of $400 \mu \mathrm{M}$ gemcitabine in the medium.

Cell transfection. The cells were transfected with $50 \mathrm{nM}$ GPRC5A siRNA (Dharmacon, Lafayette, CO, USA) or $50 \mathrm{nM}$ HuR siRNA (Ambion, Austin, TX, USA) by the reverse transfection method using the Lipofectamine RNAiMAX transfection reagent (Life Technologies). Cells transfected with only a scrambled sequence control siRNA (Dharmacon) were examined in parallel as controls. Cells were then subjected to further assays or to RNA/protein extraction after 2 days. Lipofectamine 2000 (Life Technologies) was used for transfection of the psiCHECK-2 reporter vector (Promega, Madison, WI, USA) and pcDNA-3.1 overexpression vector (Life Technologies) and for co-transfection of vectors and siRNAs.

Colony formation assay. Cells were transfected with either GPRC5A siRNA or scramble siRNA. Twenty-four hours after transfection, cells were split and seeded in six-well plates at low density $(\sim 1000$ cells per well) and treated with different doses of gemcitabine. The cells were cultured for 6 days at $37^{\circ} \mathrm{C}$ in a humidified atmosphere containing $5 \% \mathrm{CO}_{2}$. The plates were then washed with PBS and stained with crystal violet. The images of each well were scanned. The number of clones re-generated (colonies $>50$ cells each) was scored to determine the efficiency of clone formation.

In vitro cell invasion assay. The invasiveness of pancreatic cells was evaluated in 24-well Transwell chambers (Corning Inc, Corning, NY, USA), as directed by the manufacturer. Briefly, the upper and lower culture compartments of each well were separated by polycarbonate membranes (8- $\mu \mathrm{m}$ pore size), $300 \mu \mathrm{l}$ of serum-free DMEM medium were placed into the upper compartment of wells and $600 \mu \mathrm{l}$ of DMEM medium (Fisher Scientific) supplemented with $10 \%$ fetal bovine serum (Life Technologies) were placed into the lower compartment. The Transwell chambers were incubated for $14 \mathrm{~h}$ at $37^{\circ} \mathrm{C}$ in a humidified atmosphere containing $5 \% \mathrm{CO}_{2}$. Cell penetration through the membrane was detected by staining the cells on the porous membrane with crystal violet staining and quantified by counting the numbers of cells that penetrated the membrane in five microscopic fields (at $\times 200$ magnification) per filter. Each experiment was carried out in triplicate.

Cell proliferation assay. Cell proliferation was measured with CCK-8 assay. Cells were transfected with either GPRC5A siRNA or scramble siRNA, or untreated. Twenty-four hours after transfection, cells were split and seeded in 96-well plates at low density ( 1000 cells per well) and treated with different doses of gemcitabine, other drugs, or combinations of gemcitabine and Stattic (Selleckchem, Houston, TX, USA) or QNZ (Selleckchem). The cells were cultured for 6 days at $37^{\circ} \mathrm{C}$ in a humidified atmosphere containing $5 \% \mathrm{CO}_{2}$. Then the cells were incubated with $100 \mu \mathrm{l}$ of $1 \times$ CCK-8 solution for $2 \mathrm{~h}$ (Sigma-Aldrich). The absorbance was measured at $490 \mathrm{~nm}$ by using Synergy 2 Multi-Mode Microplate Reader (BioTek, Winooski, VT, USA). Data represent the average value of three wells in one experiment. Each experiment was carried out in triplicate.

Soft agar colony formation assay. Assays of colony formation in soft agar were carried out using standard methods. Briefly, $1.5 \mathrm{ml}$ bottom layers consisting of $0.5 \%$ agar medium were prepared in six-well plates by combining one volume of $5 \%$ noble agar (Difco Laboratories Inc, Detroit, MI, USA) with nine volumes of DMEM medium with $10 \%$ FBS. Cells were trypsinized, centrifuged and resuspended in $0.3 \%$ agar medium ( 1 volume of $3 \%$ noble agar and 9 volumes of DMEM medium with $10 \%$ FBS). $1 \times 10^{4}$ cells were then plated onto the previously prepared bottom layers. The cells were incubated at $37^{\circ} \mathrm{C}$ in a humidified atmosphere containing $5 \%$ $\mathrm{CO}_{2}$ for 14 days. The plates were then washed with PBS and stained with crystal violet. The images of each well were scanned and colonies were counted. 


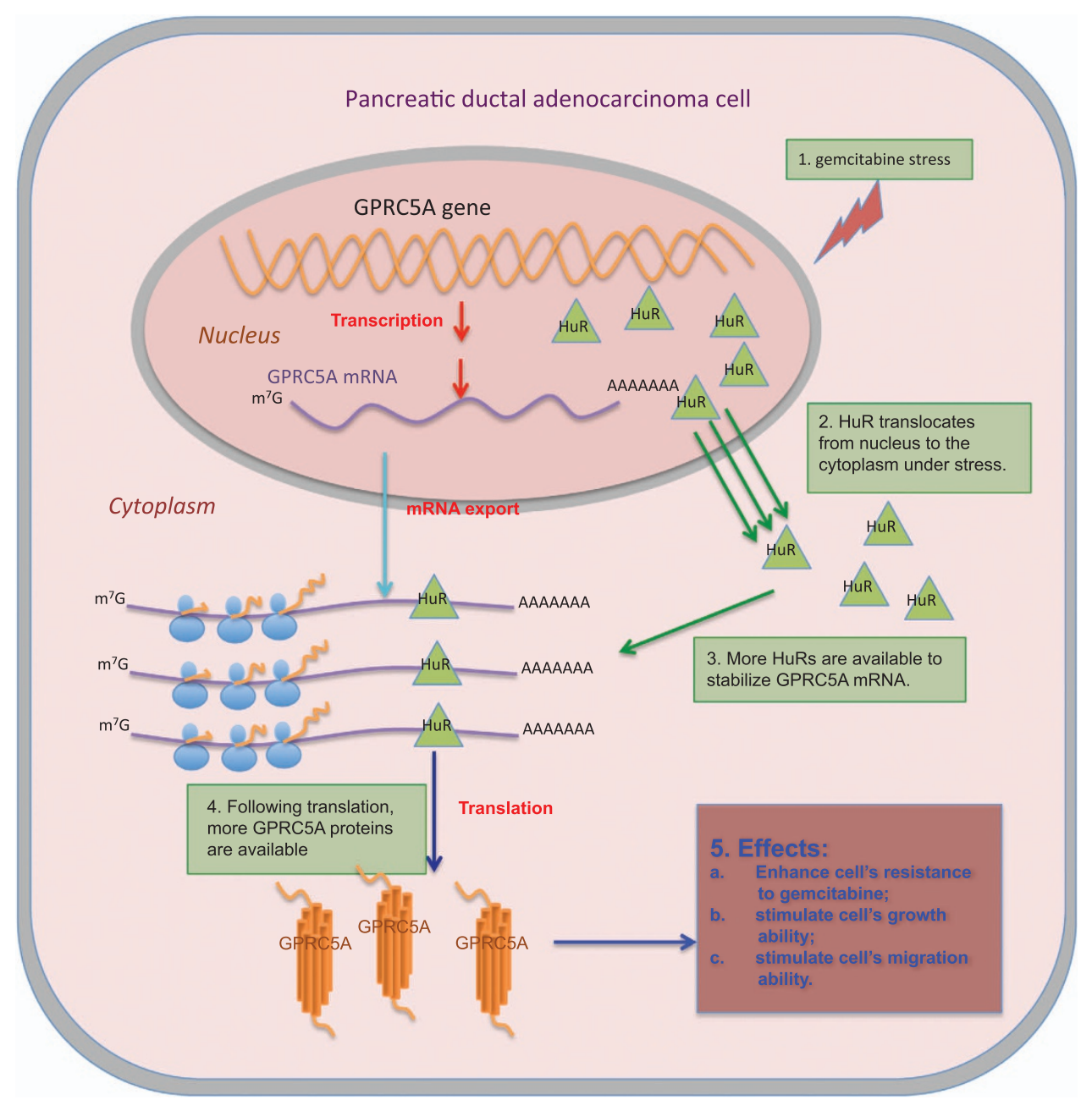

Figure 7 A schematic model for the gemcitabine treatment enhancing GPRC5A expression with the help of HuR in a pancreatic cancer cell. Upon gemcitabine stress (Step 1), HuR molecules translocate from the nucleus to the cytoplasm (Step 2). More HuR molecules are available in the cytoplasm to stabilize GPRC5A mRNA (Step 3). More GPRC5A protein is produced as a result (Step 4). GPRC5A protein enhances the pancreatic cancer cell's resistance to gemcitabine, and stimulates the pancreatic cancer cell's growth ability as well as migration ability (Step 5)

RNA isolation and real-time quantitative polymerase chain reaction analysis. Total RNA was extracted using TRIzol reagent (Life Technologies). For the detection of GPRC5A mRNA, first-strand complementary DNA was synthesized from $1000 \mathrm{ng}$ of total RNA in the presence of oligo-dT (12-18) primer (Promega) and MMLV reverse transcriptase according to the manufacturer's instructions (Promega). Human glyceraldehyde 3-phosphate dehydrogenase RNA was amplified in parallel as an internal control. Real-time qPCR was performed with SYBR Green PCR Master Mix (Life Technologies) and 20ng of templates using a StepOnePlus Real-Time PCR System (Life Technologies). All primer sequences used for GPRC5A mRNA detection are listed in Supplementary Table S1 (available Online). PCRs were performed at $95^{\circ} \mathrm{C}$ for $5 \mathrm{~min}$, followed by 40 cycles of $95^{\circ} \mathrm{C}$ for $15 \mathrm{~s}$ and $60^{\circ} \mathrm{C}$ for $1 \mathrm{~min} . \Delta \mathrm{Ct}$ was calculated by subtracting the $\mathrm{Ct}$ of $\mathrm{U} 6$ or glyceraldehyde 3-phosphate dehydrogenase mRNA from the $\mathrm{Ct}$ of the mRNA of interest. $\Delta \Delta \mathrm{Ct}$ was then calculated by subtracting the $\Delta \mathrm{Ct}$ of the negative control from the $\Delta \mathrm{Ct}$ of the sample. The fold change in mRNA was calculated according to the equation $2^{\Delta \Delta C t}$.

Computational prediction of putative HuR target sites in GPRC5A. We downloaded 12 public HuR CLIP data sets that included two HITS-CLIP and five PAR-CLIP data sets from GSE28859, one PAR-CLIP data set from GSE29779 and four PAR-CLIP data sets from GSE29943. In each case, we mapped the sequenced reads as we described previously. ${ }^{63,64}$ We note that these data sets were generated using HEK293 cells, and not pancreatic cancer cells.
Consequently, and to minimize the likelihood of working with cell-dependent regulatory interactions, ${ }^{65}$ we (a) employed stringent support thresholds; (b) used different thresholds for each data set that were adaptive to the sequencing depth in each case, ${ }^{63,64}$ and (c) considered only sites of read accumulation in GPRC5A's $3^{\prime}$ UTR that were supported by multiple among the 12 data sets.

DNA vectors. The coding region of the GPRC5A mRNA with either wild type or mutated start codon was amplified by PCR from MIA PaCa-2 CDNA using high fidelity AccuPrime Taq DNA Polymerase (Life Technologies). The fragments were inserted into the pcDNA-3.1 vector between the Nhel and Notl sites. The vectors were labeled GPRC5A WT-CDS or GPRC5A MT-CDS, respectively. The HuR overexpression vector was contributed by Dr. Jonathan Brody.

Reporter vectors. The predicted HuR-binding sites were synthesized as sense and antisense oligomers, annealed and cloned into a psiCHECK-2 vector. All primers used for these constructs are listed in Supplementary Table S1. The PIM1 psiCHECK-2 vector was contributed by Dr. Fernando Blanco and Dr. Jonathan Brody.

Luciferase assay. Each psiCHECK-2 vector containing a reporter construct was co-transfected into MIA PaCa-2 cells with HuR siRNA or HuR overexpression vector by using Lipofectamine 2000 according to the manufacturer's protocol for co-transfection of DNA and siRNAs. In parallel, each psiCHECK-2 vector containing 
a reporter construct was also co-transfected into MIA PaCa-2 cells with control siRNA or pcDNA-3.1 vector as control. Cells were harvested at 48 gemcitabine after transfection, and the Renilla and Firefly luciferase activities in the cellular lysate were assayed by using the Dual-Glo Luciferase Assay (Promega) according to the manufacturer's protocol. Light intensity for each sample was measured by using the Synergy 2 Multi-Mode Microplate Reader (BioTek), and each value from Renilla luciferase was normalized by Firefly luciferase.

Western blots. Transfected cells were lysed on ice in RIPA lysis buffer (Thermo Scientific, Waltham, MA, USA) containing $1 \times$ complete protease inhibitor (Roche, Indianapolis, IN, USA). Debris was pelletized by centrifugation at 13200 r.p.m. for $15 \mathrm{~min}$, and protein concentrations were determined using the Pierce BCA assay (Thermo Scientific). Lysates were heat-denatured at $100^{\circ} \mathrm{C}$ for $10 \mathrm{~min}$ before separation in $10 \%$ sodium dodecyl sulfate-polyacrylamide gels and transferred to nitrocellulose membrane (GE Healthcare, Mickleton, NJ, USA). Membranes were blocked with $5 \%$ bovine serum albumin (Sigma-Aldrich) in Tris-buffered saline Tween-20 buffer (10 mM Tris, pH 7.6, $150 \mathrm{mM} \mathrm{NaCl}$ and $0.1 \%$ Tween-20) and probed with primary antibody in Tris-buffered saline Tween-20 with $5 \%$ bovine serum albumin at the recommended dilutions at $4{ }^{\circ} \mathrm{C}$. Primary antibodies included GPRC5A antibody (Sigma-Aldrich), beta-actin antibody (Cell Signaling Technology, Beverly, MA, USA), HuR antibody (Santa Cruz Biotechnology, Dallas, TX, USA) and LSD1 antibody (Cell Signaling Technology). Membranes were incubated with secondary antibody (Cell Signaling Technology) diluted in Tris-buffered saline Tween-20 with $5 \%$ bovine serum albumin for 1 gemcitabine at room temperature. The signal was detected with Pierce ECL Western Blotting Substrate (Thermo Scientific) and GE ImageQuant LAS 4000 (GE Healthcare).

RIP analysis. $6.5 \times 10^{6}$ MIA PaCa-2 cells were plated to $\sim 65 \%$ confluency in $100 \mathrm{~mm}$ dish (Corning Inc., Corning, NY, USA). Cells were treated with $400 \mathrm{nM}$ gemcitabine (Selleckchem) for 0, 2, 18 and $48 \mathrm{~h}$. Cells were then trypsinized and washed with DPBS (Corning Inc.). After lysis, immunoprecipitation was performed using anti-HuR and IgG antibodies (MBL International, Woburn, MA, USA) as described in the product manuscript. The RNA was quantified by RT-qPCR using an ABI StepOnePlus qPCR System. GAPDH and dCK mRNAs were included as a loading control..$^{60}$

Statistical analysis. Statistical analysis was performed using the Excel (Microsoft, Redmond, WA, USA), SPSS (IBM, Armonk, NY, USA) and GraphPad Prism (La Jolla, CA, USA). Unless otherwise indicated, the level of significance for the difference between dose response data sets was assessed using two-way ANOVA. Other data sets were assessed using $t$-test. Data are expressed as the means \pm S.D. $P$-value $\leq 0.05$ was considered statistically significant.

Immunohistochemical analysis. The immunohistochemistry assay was performed following the standard protocol. Specifically, the antigen was retrieved with citrate buffer, $\mathrm{pH} 6.0$ at $98^{\circ} \mathrm{C}$ for $20 \mathrm{~min}$. The GPRC5A antibody HPA007928 (Sigma-Aldrich) was used in 1:300 dilution and incubated for $30 \mathrm{~min}$. The stained tissue microarrays (TMA) were analyzed in a blind manner by a pathologist (LB). Each TMA core was given a score from 0 to 3 based on the GPRC5A staining intensity of ductal cells: 0 represents no staining of ductal cells, 1 represents weak staining of ductal cells, 2 represents medium staining of ductal cells, 3 represents strong staining of ductal cells. Additionally, for each code the percentage of stained cells was noted.

\section{Conflict of Interest}

The authors declare no conflict of interest.

Acknowledgements. The work was supported in part by a Hirshberg Foundation for Pancreatic Cancer Research grant (IR) and by Institutional Funds. We thank Dr. Charles Yeo for his critical reading of the manuscript and for suggestions, and Elena Mogilyansky for making available hTERT-HPNE and MIA PaCa-2 cell stock.

1. Vincent A, Herman J, Schulick R, Hruban RH, Goggins M. Pancreatic cancer. Lancet 2011; 378: $607-620$
2. Moffitt RA, Marayati R, Flate EL, Volmar KE, Loeza SG, Hoadley KA et al. Virtual microdissection identifies distinct tumor- and stroma-specific subtypes of pancreatic ductal adenocarcinoma. Nat Genet 2015; 47: 1168-1178.

3. Brunetti O, Russo A, Scarpa A, Santini D, Reni M, Bittoni A et al. Micro-RNA in pancreatic adenocarcinoma: predictive/prognostic biomarkers or therapeutic targets? Oncotarget 2015; 6: 23323-23341.

4. Zhou H, Rigoutsos I. The emerging roles of GPRC5A in diseases. Oncoscience 2014; 1: 765-776.

5. Cheng $Y$, Lotan R. Molecular cloning and characterization of a novel retinoic acid-inducible gene that encodes a putative G protein-coupled receptor. J Biol Chem 1998; 273 : 35008-35015.

6. Wu Q, Ding W, Mirza A, Van Arsdale T, Wei I, Bishop WR et al. Integrative genomics revealed RAI3 is a cell growth-promoting gene and a novel P53 transcriptional target. J Biol Chem 2005; 280: 12935-12943.

7. Gonzales PA, Pisitkun T, Hoffert JD, Tchapyjnikov D, Star RA, Kleta R et al. Large-scale proteomics and phosphoproteomics of urinary exosomes. J Am Soc Nephrol 2009; 20: 363-379.

8. Gonzalez-Begne M, Lu B, Han X, Hagen FK, Hand AR, Melvin JE et al. Proteomic analysis of human parotid gland exosomes by multidimensional protein identification technology (MudPIT). J Proteome Res 2009; 8: 1304-1314.

9. Prunotto M, Farina A, Lane L, Pernin A, Schifferli J, Hochstrasser DF et al. Proteomic analysis of podocyte exosome-enriched fraction from normal human urine. $J$ Proteomics 2013; 82: 193-229.

10. Zougman A, Hutchins GG, Cairns DA, Verghese E, Perry SL, Jayne DG et al. Retinoic acidinduced protein 3 : identification and characterisation of a novel prognostic colon cancer biomarker. Eur J Cancer 2013; 49: 531-539.

11. Cheng L, Yang S, Yang Y, Zhang W, Xiao H, Gao H et al. Global gene expression and functional network analysis of gastric cancer identify extended pathway maps and GPRC5A as a potential biomarker. Cancer Lett 2012; 326: 105-113.

12. Dairkee SH, Sayeed A, Luciani G, Champion S, Meng Z, Jakkula LR et al. Immutable functional attributes of histologic grade revealed by context-independent gene expression in primary breast cancer cells. Cancer Res 2009; 69: 7826-7834.

13. Fujimoto J, Kadara H, Garcia MM, Kabbout M, Behrens C, Liu DD et al. G-protein coupled receptor family $\mathrm{C}$, group 5, member A (GPRC5A) expression is decreased in the adjacent field and normal bronchial epithelia of patients with chronic obstructive pulmonary disease and non-small-cell lung cancer. J Thorac Oncol 2012; 7: $1747-1754$.

14. Hofmann WK, de Vos S, Komor M, Hoelzer D, Wachsman W, Koeffler HP. Characterization of gene expression of CD34+ cells from normal and myelodysplastic bone marrow. Blood 2002; 100: 3553-3560.

15. Honda M, Yamashita T, Yamashita T, Arai K, Sakai Y, Sakai A et al. Peretinoin, an acyclic retinoid, improves the hepatic gene signature of chronic hepatitis $C$ following curative therapy of hepatocellular carcinoma. BMC Cancer 2013; 13: 191.

16. Jorissen $H$, Bektas N, Dahl E, Hartmann A, ten Haaf A, Di Fiore $S$ et al. Production and characterisation of monoclonal antibodies against RAI3 and its expression in human breast cancer. BMC Cancer 2009; 9: 200.

17. Karagiannis GS, Weile J, Bader GD, Minta J. Integrative pathway dissection of molecular mechanisms of moxLDL-induced vascular smooth muscle phenotype transformation. BMC Cardiovasc Disord 2013; 13: 4

18. Kume H, Muraoka S, Kuga T, Adachi J, Narumi R, Watanabe S et al. Discovery of colorectal cancer biomarker candidates by membrane proteomic analysis and subsequent verification using selected reaction monitoring (SRM) and tissue microarray (TMA) analysis. Mol Cell Proteomics 2014; 13: 1471-1484.

19. Liu SL, Zhong SS, Ye DX, Chen WT, Zhang ZY, Deng J. Repression of G protein-coupled receptor family $C$ group 5 member $A$ is associated with pathologic differentiation grade of oral squamous cell carcinoma. J Oral Pathol Med 2013; 42: 761-768.

20. Zhang L, Ke F, Liu Z, Bai J, Liu J, Yan S et al. MicroRNA-31 negatively regulates peripherally derived regulatory T-cell generation by repressing retinoic acid-inducible protein 3 . Nat Commun 2015; 6: 7639.

21. Nagahata T, Sato T, Tomura A, Onda M, Nishikawa K, Emi M. Identification of RAI3 as a therapeutic target for breast cancer. Endocr Relat Cancer 2005; 12: 65-73.

22. Sokolenko AP, Bulanova DR, lyevleva AG, Aleksakhina SN, Preobrazhenskaya EV, Ivantsov AO et al. High prevalence of GPRC5A germline mutations in BRCA1-mutant breast cancer patients. Int J Cancer 2014; 134: 2352-2358.

23. Subrungruanga I, Thawornkunob C, Chawalitchewinkoon-Petmitrc P, Pairojkul C, Wongkham S, Petmitrb S. Gene expression profiling of intrahepatic cholangiocarcinoma. Asian Pac J Cancer Prev 2013; 14: 557-563.

24. Tao $Q$, Fujimoto J, Men $T$, Ye X, Deng J, Lacroix $L$ et al. Identification of the retinoic acidinducible Gprc5a as a new lung tumor suppressor gene. J Natl Cancer Inst 2007; 99: 1668-1682.

25. Xin H, Wang K, Hu G, Xie F, Ouyang K, Tang X et al. Establishment and characterization of 7 novel hepatocellular carcinoma cell lines from patient-derived tumor xenografts. PLoS One 2014; 9: 885308 .

26. Zheng J, Guo X, Gao X, Liu H, Tu Y, Zhang Y. Overexpression of retinoic acid-induced protein 3 predicts poor prognosis for hepatocellular carcinoma. Clin Transl Oncol 2014; 16 : $57-63$. 
27. Danielsen JM, Sylvestersen KB, Bekker-Jensen S, Szklarczyk D, Poulsen JW, Horn H et al. Mass spectrometric analysis of lysine ubiquitylation reveals promiscuity at site level. Mol Cell Proteomics 2011; 10: M110 003590.

28. Emanuele MJ, Elia AE, Xu Q, Thoma CR, Izhar L, Leng Y et al. Global identification of modular cullin-RING ligase substrates. Cell 2011; 147: 459-474.

29. Havugimana PC, Hart GT, Nepusz T, Yang H, Turinsky AL, Li Z et al. A census of human soluble protein complexes. Cell 2012; 150: 1068-1081.

30. Hirano M, Zang L, Oka T, Ito Y, Shimada Y, Nishimura Y et al. Novel reciprocal regulation of cAMP signaling and apoptosis by orphan G-protein-coupled receptor GPRC5A gene expression. Biochem Biophys Res Commun 2006; 351: 185-191.

31. Hutchins JR, Toyoda Y, Hegemann B, Poser I, Heriche JK, Sykora MM et al. Systematic analysis of human protein complexes identifies chromosome segregation proteins. Science 2010; 328: 593-599.

32. Ji H, Wu G, Zhan X, Nolan A, Koh C, De Marzo A et al. Cell-type independent MYC target genes reveal a primordial signature involved in biomass accumulation. PLoS One 2011; 6: e26057.

33. Kim W, Bennett EJ, Huttlin EL, Guo A, Li J, Possemato A et al. Systematic and quantitative assessment of the ubiquitin-modified proteome. Mol Cell 2011; 44: 325-340.

34. Lee KA, Hammerle LP, Andrews PS, Stokes MP, Mustelin T, Silva JC et al. Ubiquitin ligase substrate identification through quantitative proteomics at both the protein and peptide levels J Biol Chem 2011; 286: 41530-41538.

35. Meierhofer D, Wang X, Huang L, Kaiser P. Quantitative analysis of global ubiquitination in HeLa cells by mass spectrometry. J Proteome Res 2008; 7: 4566-4576.

36. Povlsen LK, Beli P, Wagner SA, Poulsen SL, Sylvestersen KB, Poulsen JW et al. Systemswide analysis of ubiquitylation dynamics reveals a key role for PAF15 ubiquitylation in DNAdamage bypass. Nat Cell Biol 2012; 14: 1089-1098.

37. Wagner SA, Beli P, Weinert BT, Nielsen ML, Cox J, Mann M et al. A proteome-wide quantitative survey of in vivo ubiquitylation sites reveals widespread regulatory roles. Mol Cell Proteomics 2011; 10: M111 013284.

38. Ye X, Lotan R. Potential misinterpretation of data on differential gene expression in normal and malignant cells in vitro. Brief Funct Genomic Proteomic 2008; 7: 322-326.

39. Ye X, Tao $Q$, Wang $Y$, Cheng $Y$, Lotan R. Mechanisms underlying the induction of the putative human tumor suppressor GPRC5A by retinoic acid. Cancer Biol Ther 2009; 8: 951-962.

40. Oppermann FS, Grundner-Culemann K, Kumar C, Gruss OJ, Jallepalli PV, Daub H. Combination of chemical genetics and phosphoproteomics for kinase signaling analysis enables confident identification of cellular downstream targets. Mol Cell Proteomics 2012; 11: 0111012351

41. Olsen JV, Vermeulen M, Santamaria A, Kumar C, Miller ML, Jensen LJ et al. Quantitative phosphoproteomics reveals widespread full phosphorylation site occupancy during mitosis. Sci Signal 2010; 3: ra3.

42. Shiromizu T, Adachi J, Watanabe S, Murakami T, Kuga T, Muraoka S et al. Identification of missing proteins in the neXtProt database and unregistered phosphopeptides in the PhosphoSitePlus database as part of the Chromosome-centric Human Proteome Project. J Proteome Res 2013; 12: 2414-2421.

43. Dephoure N, Zhou C, Villen J, Beausoleil SA, Bakalarski CE, Elledge SJ et al. A quantitative atlas of mitotic phosphorylation. Proc Natl Acad Sci USA 2008; 105: 10762-10767.

44. Deng J, Fujimoto J, Ye XF, Men TY, Van Pelt CS, Chen YL et al. Knockout of the tumor suppressor gene Gprc5a in mice leads to NF-kappaB activation in airway epithelium and promotes lung inflammation and tumorigenesis. Cancer Prev Res 2010; 3: 424-437.

45. Ma WJ, Cheng S, Campbell C, Wright A, Furneaux H. Cloning and characterization of HuR, a ubiquitously expressed Elav-like protein. J Biol Chem 1996; 271: 8144-8151.

46. Ma WJ, Furneaux H. Localization of the human HuR gene to chromosome 19p13.2. Hum Genet 1997; 99: 32-33.

47. Scheiba RM, de Opakua Al, Diaz-Quintana A, Cruz-Gallardo I, Martinez-Cruz LA Martinez-Chantar ML et al. The C-terminal RNA binding motif of HuR is a multi-functional domain leading to HuR oligomerization and binding to U-rich RNA targets. RNA Biol 2014; 11: $1250-1261$.

48. Lopez de Silanes I, Zhan M, Lal A, Yang X, Gorospe M. Identification of a target RNA motif for RNA-binding protein HuR. Proc Natl Acad Sci USA 2004; 101: 2987-2992.
49. Mukherjee N, Corcoran DL, Nusbaum JD, Reid DW, Georgiev S, Hafner M et al. Integrative regulatory mapping indicates that the RNA-binding protein HuR couples pre-mRNA processing and mRNA stability. Mol Cell 2011; 43: 327-339.

50. Brennan CM, Steitz JA. HuR and mRNA stability. Cell Mol Life Sci 2001; 58: 266-277.

51. Doller A, Pfeilschifter J, Eberhardt W. Signalling pathways regulating nucleo-cytoplasmic shuttling of the mRNA-binding protein HuR. Cell Signal 2008; 20: 2165-2173.

52. Chang SH, Hla T. Post-transcriptional gene regulation by HuR and microRNAs in angiogenesis. Curr Opin Hematol 2014; 21: 235-240.

53. Meisner NC, Filipowicz W. Properties of the regulatory RNA-binding protein $\mathrm{HuR}$ and its role in controlling miRNA repression. Adv Exp Med Biol 2010; 700: 106-123.

54. Wilusz CJ, Wilusz J. HuR and translation - the missing linc(RNA). Mol Cell 2012; 47: 495-496.

55. Kotta-Loizou I, Giaginis C, Theocharis S. Clinical significance of HuR expression in human malignancy. Med Oncol 2014; 31: 161

56. Costantino CL, Witkiewicz AK, Kuwano Y, Cozzitorto JA, Kennedy EP, Dasgupta A et al. The role of $\mathrm{HuR}$ in gemcitabine efficacy in pancreatic cancer: HuR Up-regulates the expression of the gemcitabine metabolizing enzyme deoxycytidine kinase. Cancer Res 2009; 69 : 4567-4572.

57. Klijn C, Durinck S, Stawiski EW, Haverty PM, Jiang Z, Liu $\mathrm{H}$ et al. A comprehensive transcriptional portrait of human cancer cell lines. Nat Biotechnol 2015; 33 306-312.

58. R Core Team R: A Language and Environment for Statistical Computing. R Foundation for Statistical Computing: Vienna, Austria, 2014

59. Zhou H, Rigoutsos I. MiR-103a-3p targets the 5' UTR of GPRC5A in pancreatic cells. Rna 2014; 20: 1431-1439.

60. Pineda DM, Rittenhouse DW, Valley CC, Cozzitorto JA, Burkhart RA, Leiby B et al. HuR's post-transcriptional regulation of Death Receptor 5 in pancreatic cancer cells. Cancer Bio Ther 2012; 13: 946-955.

61. Lal S, Burkhart RA, Beeharry N, Bhattacharjee V, Londin ER, Cozzitorto JA et al. HuR posttranscriptionally regulates WEE1: implications for the DNA damage response in pancreatic cancer cells. Cancer Res 2014; 74: 1128-1140.

62. Blanco FF, Jimbo M, Wulfkuhle J, Gallagher I, Deng J, Enyenihi L et al. The mRNA-binding protein HuR promotes hypoxia-induced chemoresistance through posttranscriptional regulation of the proto-oncogene PIM1 in pancreatic cancer cells. Oncogene 2015; 35 2529-2541.

63. Clark PM, Loher P, Quann K, Brody J, Londin ER, Rigoutsos I. Argonaute CLIP-Seq reveals miRNA targetome diversity across tissue types. Sci Rep 2014; 4: 5947

64. Londin E, Loher P, Telonis AG, Quann K, Clark P, Jing Y et al. Analysis of 13 cell types reveals evidence for the expression of numerous novel primate- and tissue-specific microRNAs. Proc Natl Acad Sci USA 2015; 112: E1106-E1115.

65. Quann K, Jing Y, Rigoutsos I. Post-transcriptional regulation of BRCA1 through its coding sequence by the miR-15/107 group of miRNAs. Front Genet 2015; 6: 242.

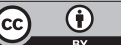

Cell Death and Disease is an open-access journal published by Nature Publishing Group. This work is licensed under a Creative Commons Attribution 4.0 International License. The images or other third party material in this article are included in the article's Creative Commons license, unless indicated otherwise in the credit line; if the material is not included under the Creative Commons license, users will need to obtain permission from the license holder to reproduce the material. To view a copy of this license, visit http://creativecommons.org/licenses/by/4.0/

(C) The Author(s) 2016

Supplementary Information accompanies this paper on Cell Death and Disease website (http://www.nature.com/cddis) 